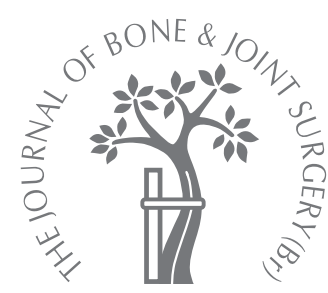

B. Kocaoglu, I. Agir, U. Nalbantoglu, M. Karahan, M. Türkmen

From Acibadem University, Faculty of Medicine, Turkey

\title{
Effect of Mitomycin-C on post-operative adhesions in tendon surgery
}

\author{
AN EXPERIMENTAL STUDY IN RATS
}

\footnotetext{
B. Kocaoglu, MD, Orthopaedic Surgeon U. Nalbantoglu, MD, Associate Professor

M. Türkmen, MD, Professor Department of Orthopaedics and Traumatology

Acibadem University, Faculty of Medicine, Soyak Eureka, Al blok D:43, Soganlik, 34880, Kartal, Istanbul, Turkey.

I. Agir, MD, Orthopaedic Surgeon

M. Karahan, MD, Professor Department of Orthopedics and Traumatology

Marmara University, Faculty of Medicine, Barbaros mah. Kentplus I. Etap, Cl blok D:52, 34760, Yenisahra, Istanbul, Turkey.
}

Correspondence should be sent to $\mathrm{Dr}$ B. Kocaoglu; e-mail: bariskocaoglu@gmail.com

(c)2010 British Editorial Society of Bone and Joint Surgery doi:10.1302/0301-620X.92B6. $23534 \$ 2.00$

$J$ Bone Joint Surg $[\mathrm{Br}]$ 2010;92-B:889-93. Received 1 October 2009 Accepted after revision 26 January 2010

We investigated the effect of mitomycin- $C$ on the reduction of the formation of peritendinous fibrous adhesions after tendon repair. In $\mathbf{2 0}$ Wistar albino rats the tendo Achillis was cut and repaired using a modified Kessler technique. The rats were divided into two equal groups. In group 1, an injection of mitomycin-C was placed between the tendon and skin of the right leg. In group 2, an identical volume of sterile normal saline was injected on the left side in a similar fashion. All the rats received mitomycin-C or saline for four weeks starting from the day of operation. The animals were killed after $\mathbf{3 0}$ days. The formation of peritendinous fibrous tissue, the inflammatory reaction and tendon healing were evaluated. The tensile strength of the repaired tendons was measured biomechanically. Microscopic evidence of the formation of adhesions and inflammation was less in group 1. There was no significant difference in the tensile load required to rupture the repaired tendons in the two groups.

Mitomycin-C may therefore provide a simple and inexpensive means of preventing postoperative adhesions.

In spite of improvements in surgical technique and post-operative mobilisation, the result of surgery on flexor tendons is still highly unpredictable because of the formation of adhesions. ${ }^{1}$ Many different approaches have been tried to prevent their formation. Advances in techniques of suturing, reconstruction of the tendon sheath and early controlled mobilisation have led to a decrease in their formation, ${ }^{1-4}$ but the use of these methods has not solved the problem entirely.

The control of excessive scar formation is essential to restore the functional integrity of a healing tendon after surgery. The main techniques for reducing adhesions without adversely affecting the healing process are mechanical and biochemical. Mechanical barriers such as nylon, cellophane, teflon and polyethylene have all been used in an attempt to form a pseudosheath and thereby prevent fibrous in-growth. The results have been disappointing. ${ }^{2,5-7}$

Application of a biochemical agent to prevent fibrosis may eliminate fibrotic adhesions. Numerous substances have been used to prevent the formation of fibrosis with varying effectiveness, but reports of the use of mitomycin-C (MMC) in animal models have shown it to be effective in preventing the postoperative formation of intraperitoneal adhe- sions by inhibiting the proliferation of fibroblasts. ${ }^{8-10}$ It is active against all cell lines and has been used topically in man and successfully in ophthalmic surgery and in otorhinolaryngology. ${ }^{7,8}$ We could not find any study on the effect of MMC in the prevention of adhesions in tendon surgery. ${ }^{11-13}$ Our aim therefore was to investigate the effect of MMC on the formation of peritendinous fibrotic adhesions after tendon repair. We hypothesised that the application of MMC to the tendon sheath in the rat would reduce their formation.

\section{Materials and Methods}

We used 20 female Wistar albino rats weighing between $350 \mathrm{~g}$ and $450 \mathrm{~g}$. Permission was given to use laboratory animals by the Research Ethics Commission for animal experiments. Animal care complied with the guidelines of our institution, the National Institutes of Health and national law on the care and use of laboratory animals.

The animals were anaesthetised with an intraperitoneal injection of pentothal $(50 \mathrm{mg} / \mathrm{kg})$. A pre-operative dose of intramuscular cefazolin sodium $(0.1 \mathrm{mg} / \mathrm{kg})$ was given for prophylaxis against infection. The rat was positioned prone on the operating table and the same surgeon (BK) performed the operations using an identical technique. Both hind limbs were shaved. 


\begin{tabular}{|c|c|}
\hline \multicolumn{2}{|l|}{ Quality } \\
\hline 0 & No apparent adhesions \\
\hline 1 & A number of scattered filaments \\
\hline 2 & A large number of filaments \\
\hline 3 & Countless filaments \\
\hline \multicolumn{2}{|l|}{ Quality } \\
\hline 0 & No apparent adhesions \\
\hline 1 & Regular, elongated, fine, filamentous \\
\hline 2 & $\begin{array}{l}\text { Irregular, mixed, shortened, } \\
\text { filamentous }\end{array}$ \\
\hline 3 & Dense, not filamentous \\
\hline \multicolumn{2}{|c|}{ Grading of adhesions } \\
\hline 0 & None \\
\hline 1 to 2 & Slight \\
\hline 3 to 4 & Moderate \\
\hline 5 to 6 & Severe \\
\hline
\end{tabular}

The surgical field was prepared with povidone-iodine soap and solution and draped in an aseptic fashion. A posterior longitudinal skin incision of $2 \mathrm{~cm}$ was made in both legs sequentially. After dissection of the skin and subcutaneous tissues, the tendo Achillis was exposed and a full-thickness transverse tenotomy was made with a number 15 blade, $0.5 \mathrm{~cm}$ proximal to its insertion. The ruptured tendons were repaired by a modified Kessler technique using 5/0 monofilament polypropylene (Prolene; Ethicon, Somerville, New Jersey) sutures. The tendon sheaths were not repaired.

The animals were divided into two equal groups. In group 1, an injection of MMC $(0.2 \mathrm{ml}$ with $0.08 \mathrm{mg})$ was placed between the tendon and the skin of the right leg with a $27 \mathrm{G}$ dental needle, just after the final skin closure. In group 2, an identical volume of sterile normal saline was injected on the left side in a similar fashion. All the rats received four weekly injections of MMC $(0.2 \mathrm{ml}$ with $0.08 \mathrm{mg}$ ) and saline starting from the day of operation. External immobilisation was not used. The rats were fed a standard diet, kept in a 12-hour night-day cycle and allowed water in their cages without restriction.

The animals were killed after 30 days by a lethal dose of pentobarbital $(150 \mathrm{mg} / \mathrm{kg})$ and both hind limbs were disarticulated at the knee. The criteria described by Tang, Shi and Zhang ${ }^{14}$ were used for the quantitative macroscopic evaluation of the peritendinous adhesions. This was performed subjectively by one of the investigators (IA) in a blinded fashion to eliminate any bias. The values for each group were compared with the control group according to an arbitrary scale (grade 0 , free sliding; grade 1 , minimal restriction of the range of movement to $50 \%$ to $80 \%$ of the opposite side; grade 2, moderate restriction of movement of less than $50 \%$; grade 3 , excessive restriction to less than $20 \%$; and grade 4 , no sliding) ${ }^{1}$

Histological assessment. The tendons of both limbs of ten animals (20 limbs) were collected and fixed in 10\% neutral buffered formalin, dehydrated in an ascending alcohol series and embedded in paraffin wax. Sections approximately $7 \mu \mathrm{m}$ thick were stained by Masson's trichrome stain (Fisher Scientific Company, Middletown, Ohio). The stained sections were evaluated microscopically (Olympus BH-2, Tokyo, Japan) by two independent observers (IA, VA) who were blinded, for the presence and density of fibrosis, the vascularity at the fibrosis and for the extent of normal tendon healing. ${ }^{1,4}$ The criteria of Tang et a ${ }^{14}$ were used to evaluate both the development of peritendinous fibrous tissue (Table I) and tendon healing (Table II). Those of Moran et $\mathrm{al}^{15}$ were used to assess peritendinous inflammatory reaction (Table III).

Biomechanical testing. This was performed in both limbs of ten animals on the day of their death. Both the right and the left tendons were dissected from the distal and proximal insertions. The tensile strength of the repaired tendons was measured using the Instron device (Model 5900, Instron Experimental Instruments, Norwood, Massachusetts). The ultimate breaking strengths of the tendons reflected the differences in the healing between the groups.

The distal end of the tendon was grasped with the compressive clamp and the proximal end was secured by the grip of the traction device. The tensile strength of the repaired tendon was assessed using longitudinally applied loads. The Instron device pulled the tendon at a constant rate of $110 \mathrm{~mm} / \mathrm{s}$, gradually increasing the tensile load $(\mathrm{kg} \mathrm{cm})$ until the tendon ruptured. Measurements of the tensile load were recorded on an x-y recorder (Bluehill software, Norwood, Massachusetts).

Statistical analysis. Statistical significance of the results of grading of the adhesions, tendon healing and comparisons of the groups were determined using the Mann-Whitney $U$ test. The results if the tensile strength of the repaired tendons were evaluated using Student's $t$-test. A p-value $\leq 0.05$ was considered to be significant. All evaluations were performed using the Statistical Package for Social Sciences (SPSS Inc., Chicago, Illinois).

\section{Results}

All the animals completed the experimental period successfully and no tendon rupture was observed. They walked in their cages without any difficulty. No wound dehiscence, wound infection or tissue necroses occurred. Macroscopically, the amount of scar tissue present was lower in group 1 (mean 0.5 (0 to 1$) \pm 0.5$; Fig. 1a) compared with group 2 (mean 2.1 (1 to 4$) \pm 0.7$; Fig. 1b). This difference was statistically significant $(\mathrm{p}=0.026)$.

Microscopic evidence of the formation of adhesions was less in group 1 and the difference between the groups was statistically significant $(\mathrm{p}<0.01$; Table IV).

In group 1 eight of ten tendons and in group 2, three of ten tendons showed no inflammatory reaction $(\mathrm{p}<0.01$; Table IV).

In group 1 ten tendons, and in group 2, one tendon showed excellent-to-good healing which was statistically 
Table II. Details of the grading system for tendon healing, according to the criteria of Tang et al ${ }^{14}$

\begin{tabular}{ll}
\hline Grade & Description \\
\hline Excellent & $\begin{array}{l}\text { Continuity of tendon was re-established and the epitenon was smooth } \\
\text { Regular intratendinous collagen bundles, but the epitenon was destroyed by } \\
\text { adhesions }\end{array}$ \\
Fair & $\begin{array}{l}\text { Irregular intratendinous collagen bundles, and partly interrupted by adhesions } \\
\text { Poor }\end{array}$ \\
\hline
\end{tabular}

Table III. Details of the grading system for the evaluation of the peritendinous inflammatory reaction according to the criteria of Moran et $\mathrm{al}^{15}$

\begin{tabular}{ll}
\hline Grade & Inflammatory reaction \\
\hline 0 & No reaction \\
1 & Minimal leukocyte infiltration into fibro-osseous tendon sheath \\
2 & Leukocyte infiltration in synovium and epitenon \\
3 & Leukocyte infiltration in synovium and endotenon \\
4 & Diffuse leukocyte infiltration \\
\hline
\end{tabular}

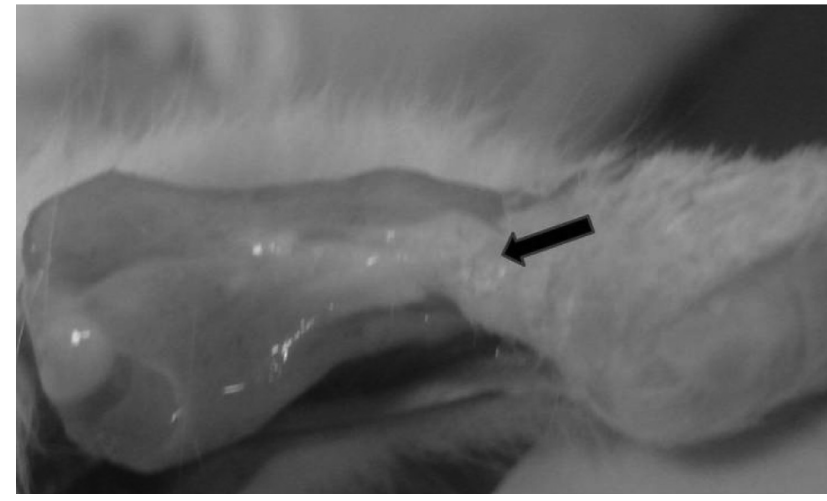

Fig. 1a

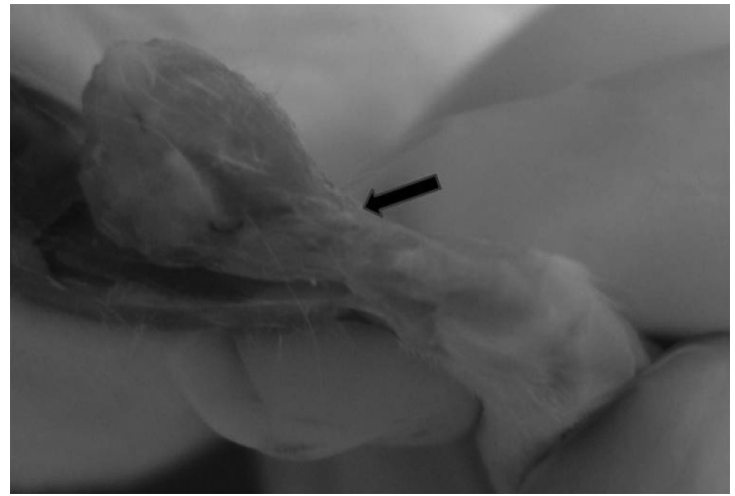

Fig. $1 b$

Photographs showing a) the tendon repair after application of mitocytin-C showing a smooth surface and less fibrous tissue formation. The arrow shows the site of the repair and b) a control tendon showing an irregular and rough surface with an increase in the formation of fibrous tissue. The arrow shows the increase in the fibrous tissue and suture material at the site of the repair.

significant $(\mathrm{p}<0.001$; Table V). Comparing the tendons of both groups, the healing of group 1 had a better morphology (Fig. 2). In group 2, the tendons showed a disordered arrangement of collagen fibres and a prominent increase in vascularity. The regular, well-ordered appearance of the collagen fibres had been disrupted and the characteristic array structure had been lost (Fig. 3). The MMC tendon group showed healing with nearly regular collagen fibres keeping the hierarchical structure. The tensile load values are summarised in Table VI. There was no significant difference in the tensile load required to rupture the repaired tendon in the groups $(\mathrm{p}=0.567)$.

\section{Discussion}

At the cellular level fibroblasts are responsible for most of the adhesions between the tendon and flexor sheath. Originally, it was thought that fibroblasts migrated inwards from the tendon sheath to the site of injury, creating an essential and external means of repair. ${ }^{16}$ The biological process of healing has both an intrinsic mechanism of repair with resident tenocytes and an extrinsic process with fibroblasts and inflammatory cells from the tendon sheath. Recently, Chang et al ${ }^{17}$ highlighted the dual mechanism of the biological process of tendon repair and an additional biochemical mechanism which may influence excessive scar formation. They noted that extrinsic healing mostly formed excessive scar formation. ${ }^{17}$ For that reason, to avoid the extent of the formation of adhesions in the healing area, separation of the healing tendon from its surrounding tissues has been advocated. Mechanical barriers have been designed for this purpose. ${ }^{1,3,4}$ However, these isolation techniques have generally been unsuccessful. Some caused impairment of the healing process and some stimulated a severe inflammatory response.

If a biochemical agent could be directed against the extrinsic fibroblasts, it would be extremely effective in 
Table IV. Histopathological evaluation of peritendinous adhesions and the inflammatory reaction in both groups

\begin{tabular}{|c|c|c|c|c|c|}
\hline & \multicolumn{5}{|l|}{ Grade } \\
\hline & None & Slight & Moderate & Severe & Total \\
\hline \multicolumn{6}{|c|}{ Adhesion status of tendons } \\
\hline Group 1 & 7 & 3 & 0 & 0 & 10 \\
\hline \multirow[t]{3}{*}{ Group 2} & 0 & 2 & 5 & 3 & 10 \\
\hline & \multicolumn{5}{|l|}{ Grade } \\
\hline & 0 & 1 & 2 & 3 & 4 \\
\hline \multicolumn{6}{|c|}{ Peritendinous inflammatory reaction } \\
\hline Group 1 & 8 & 1 & 1 & 0 & 0 \\
\hline Group 2 & 3 & 3 & 2 & 1 & 1 \\
\hline
\end{tabular}

Table V. Details of the histopathological evaluation of the quality of tendon healing

\begin{tabular}{clllll}
\hline & \multicolumn{3}{l}{ Quality of healing } & & \\
\cline { 2 - 5 } & Excellent & Good & Fair & Poor & Total \\
\hline $\begin{array}{c}\text { Groups } \\
1\end{array}$ & 9 & 1 & 0 & 0 & 10 \\
2 & 1 & 1 & 5 & 3 & 10 \\
\hline
\end{tabular}

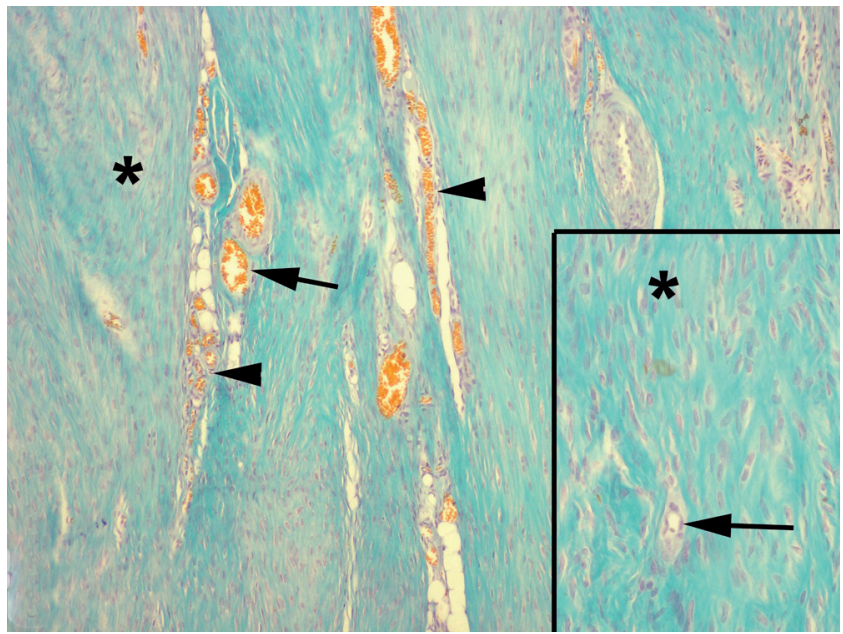

Fig. 2

Photomicrograph of the tendon repair of a right limb after application of mitomycin-C showing healing with nearly regular collagen fibres keeping the hierarchical structure with minimal peritendinous fibrosis. The large arrow shows the suture material and repair site. The two small arrows show fibrous adhesions (positive trichrome-positive fibres in brown) at the epitenon. The collagen fibres are blue (Masson's trichrome stain $\times$ $100)$. The inset on the right shows cells which had regular isogenous alignment and morphology $(\times 200) .{ }^{*}$, shows the magnified area.

preventing adhesions and improving the excursion of the tendon. MMC could be such an agent. It is produced by Streptomyces cuspidatus and was used initially as an aminoglycoside antibiotic. It is an antineoplastic agent which has been used safely in man since $1983 .{ }^{8-10}$ Its mechanism of action is through cross-linking of DNA. MMC has been investigated for the last 20 years for its ability to

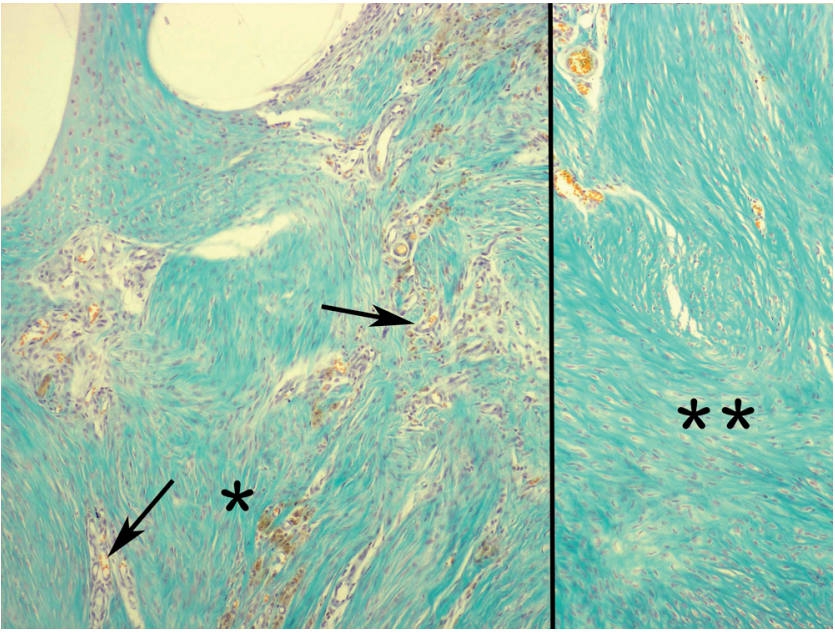

Fig. 3

Photomicrograph of the tendon of a left limb of the control group showing that the regular, well-ordered appearance of the collagen fibres has been disrupted and the characteristic array structure has been lost. The collagen fibres are in blue. The arrows show an increase in fibrous adhesions (trichrome-positive fibres in brown) (Masson's trichrome stain $x$ 100 (left), $\times 200$ (right)). **, shows the magnified area).

reduce scar formation. Its proposed mechanism of action is by the prevention of the proliferation of fibroblasts, a major step in scar formation, by blocking the synthesis of DNA by cross-linking DNA. ${ }^{8-10}$ In animal models, it has been found to be effective in preventing the post-operative re-formation of intraperitoneal adhesions by inhibiting fibroblastic proliferation. ${ }^{18}$ The antiproliferative effect of MMC on cultured human fibroblasts has been observed at lower concentrations, whereas cytocidal activity against these cells was seen at higher concentrations, at which the drug started to inhibit RNA and protein synthesis. ${ }^{19}$

In our study MMC was shown to consistently reduce post-operative tendon adhesions. Wistar rats are an appropriate model, because the tendo Achillis is suitable for surgical manipulation. ${ }^{1,4}$ The gross range of movement was used as an indirect measure of the level of formation of tendon adhesions, ${ }^{1,4}$ but was not thought to be an adequate 
Table VI. Details of the biomechanical strength of the repair in both groups

\begin{tabular}{lll}
\hline \multirow{2}{*}{ Number of rats } & \multicolumn{2}{l}{ Mean tensile load value (kg/cm) } \\
\cline { 2 - 3 } & Group 1 & Group 2 \\
\hline 1 & 22.2 & 11.4 \\
2 & 14.5 & 23.5 \\
3 & 24.8 & 9.8 \\
4 & 23.1 & 24.7 \\
5 & 36.6 & 12.9 \\
6 & 24.3 & 29.0 \\
7 & 25.6 & 39.8 \\
8 & 37.8 & 36.7 \\
9 & 17.8 & 35.6 \\
10 & 32.3 & 22.4 \\
Mean force (range) & 25.9 (14.5 to 37.8) & 24.6 (9.8 to 39.8) \\
\hline
\end{tabular}

* the difference between the two groups, $\mathrm{p}=0.657$

indicator for assessing the level of tendon adhesion in this model. In our study the experimental use of MMC in tendon healing resulted in the decreased formation of adhesions both macro- and microscopically. This could be the result of a decrease in fibroblast proliferation. ${ }^{8-10}$ We used low doses of MMC, but the role of the concentration and molecular weight of MMC in this situation is not clear and further investigation using a dose-titration evaluation scheme is required.

MMC mostly affected the extrinsic pathway and inhibited excessive scar formation around the tendon. It did not affect the intrinsic healing. Comparing the tendons of both groups, the healing of tendons exposed to MMC had a better morphology because this group was not affected by excessive scar formation. In the control group, the tendons had a disordered arrangement of collagen fibres and a prominent increase in vascularity and inflammation. MMC also appeared to decrease the inflammatory reaction compared with the control group.

MMC did not affect either healing or the tendon strength. The tensile load required to cause rupture of the repaired tendons did not differ in the two groups. We only measured biochemical testing at four weeks because our aim was just to evaluate the final outcome of the effect of $\mathrm{MMC}$ on tendon healing.

Our study has shown that weekly application of MMC to the tendon sheath of the rat significantly reduced the formation of peritendinous fibrous adhesions. MMC may provide a simple and inexpensive means of preventing the formation of post-operative adhesions. Further study needs to be carried out to attempt to elucidate the mechanism of action of MMC on the prevention of the formation of adhesions in order to delineate the ideal dose required and to examine possible adverse effects of its use.

No benefits in any form have been received or will be received from a commercial party related directly or indirectly to the subject of this article.

\section{References}

1. Tuncay I, Ozbek H, Atik B, Ozen S, Akpinar F. Effects of hyaluronic acid on postoperative adhesion of tendo calcaneus surgery: an experimental study in rats. $J$ Foot Ankle Surg 2002;41:104-8.

2. Ișik S, Oztürk S, Gürses S, et al. Prevention of restrictive adhesions in primary tendon repair by HA-membrane: experimental research in chickens. Br J Plast Surg 1999;52:373-9.

3. Liu Y, Skardal A, Shu XZ, Prestwich GD. Prevention of peritendinous adhesions using a hyaluronan-derived hydrogel film following partial-thickness flexor tendon injury. J Orthop Res 2008;26:562-9.

4. Temiz A, Ozturk C, Bakunov A, Kara K, Kaleli T. A new material for prevention of peritendinous fibrotic adhesions after tendon repair: oxidised regenerated cellulose (Interceed), an absorbable adhesion barrier. Int Orthop 2008;32:389-94.

5. Ferguson RE, Rinker $\mathbf{B}$. The use of a hydrogel sealant on flexor tendon repairs to prevent adhesion formation. Ann Plast Surg 2006;56:54-8

6. Güdemez E, Ekșioğlu F, Korkusuz $\mathbf{P}$, et al. Chondroitin sulfate-coated polyhydroxyethyl methacrylate membrane prevents adhesion in full-thickness tendon tears of rabbits. J Hand Surg Am 2002;27:293-306.

7. Frykman E, Jacobsson S, Widenfalk B. Fibrin sealant in prevention of flexor tendon adhesions: an experimental study in the rabbit. J Hand Surg Am 1993;18:68-75.

8. Aydin E, Uckan S, Ozdemir BH, Uyar P. Mitomycin C effect on fibrous adhesions of rabbit temporomandibular joint. Otolaryngol Head Neck Surg 2005;133:672-6.

9. Chen PL, Chen WY, Lu DW. Evaluation of mitomycin $C$ in reducing postoperative adhesions in strabismus surgery. J Ocul Pharmacol Ther 2005;21:406-10.

10. Lee JY, Stenzel W, Löhr M, et al. The role of mitomycin $C$ in reducing recurrence of epidural fibrosis after repeated operation in a laminectomy model in rats. J Neurosurg Spine 2006;4:329-33

11. Ates U, Ata B, Ortakuz S, Seyhan A, Urman B. Prevention of adhesion formation following ovarian surgery in a standardized animal model: comparative study of Interceed and double layer Surgicell. J Obstet Gynaecol Res 2008;34:12-17.

12. Karakurum G, Buyukbebeci O, Kalender M, Gulec A. Seprafilm interposition for preventing adhesion formation after tenolysis: an experimental study on the chicken flexor tendons. J Surg Res 2003;113:195-200.

13. Ozgenel GY. The effects of a combination of hyaluronic and amniotic membrane on the formation of peritendinous adhesions after flexor tendon surgery in chickens. $J$ Bone Joint Surg [Br] 2004;86-B:301-7.

14. Tang JB, Shi D, Zhang QG. Biomechanical and histologic evaluation of tendon sheath management. J Hand Surg Am 1996;21:900-8.

15. Moran SL, Ryan CK, Orlando GS, Pratt CE, Michalko KB. Effects of 5-fluorouracil on flexor tendon repair. J Hand Surg Am 2000;25:242-51.

16. Peacock EE Jr. Biological principles in the healing of long tendons. Surg Clin North Am 1965;45:461-76

17. Chang J, Most D, Stelnicki E, et al. Gene expression of transforming growth factor beta-1 in rabbit zone II flexor tendon wound healing: evidence for dual mechanisms of repair. Plast Reconstr Surg 1997;100:937-44.

18. Cubukçu A, Alponat A, Gönüllü NN. Mitomycin-C prevents reformation of intraabdominal adhesions after adhesiolysis. Surgery 2002;131:81-4.

19. Spector JE, Werkhaven JA, Spector NC, et al. Preservation of function and histologic appearance in the injured glottis with topical mitomycin-C. Laryngoscope 1999;109:1125-9. 